\title{
International Conference «Organometallic and Coordination Chemistry: Achievements and Challenges» (VI Razuvaev Lectures) September 18-23, 2015
}

\author{
G. A. Razuvaev Institute of Organometallic Chemistry of RAS, \\ Division of Chemistry and Materials Science of RAS \\ N. I. Lobachevsky Nizhny Novgorod State University \\ Nizhny Novgorod Science Center of RAS
}

The conference will take place on a board of comfortable four-decker ship «Mikhail Frunze» running along Volga river according with itinerary Nizhny Novgorod-Kazan-Samara-Cheboksary-Nizhny Novgorod. The departure from Nizhny Novgorod is on Friday, September 18 at 23-55, the arrival to Nizhny Novgorod is on Thursday, September 23 at 17-00.

\section{Organizing Committee:}

Prof. Gleb A. Abakumov (Nizhny Novgorod)

Prof. Vladimir K. Cherkasov (Nizhny Novgorod)

Dr. Klara G. Shalnova (Nizhny Novgorod)

\author{
Chairman \\ Vice Chairman \\ Scientific Secretary
}

Prof. Sergey M. Aldoshin (Chernogolovka)

Prof. Irina P. Beletskaya (Moscow)

Prof. Mikhail N. Bochkarev (Nizhny Novgorod)

Prof. Vladimir I. Bregadze (Moscow)

Prof. Yury N. Bubnov (Moscow)

Prof. Anatoly L. Buchachenko (Moscow)

Prof. Evgeny V. Chuprunov (Nizhny Novgorod)

Prof. Viktor A. Dodonov (Nizhny Novgorod)

Prof. Vladimir P. Fedin (Novosibirsk)

Prof. Igor L. Fedushkin (Nizhny Novgorod)

Prof. Dmitry F. Grishin (Nizhny Novgorod)
Prof. Mikhail P. Egorov (Moscow)

Prof. Igor L. Eremenko (Moscow)

Prof. Alexandr I. Konovalov (Kazan)

Prof. Ilya I. Moiseev (Moscow)

Prof. Aziz M. Muzafarov (Moscow)

Prof. Vladimir M. Novotortsev (Moscow)

Prof. Viktor I. Ovcharenko (Novosibirsk)

Prof. Elena S. Shubina (Moscow)

Prof. Oleg G. Sinyashin (Kazan)

Prof. Alexandr A. Trifonov (Nizhny Novgorod)

All modern advances and new trends in organometallic and coordination chemistry will be presented.

Sessions will include the following topics:

- Transition metals, lanthanides, main group elements

- Coordination and bioinorganic chemistry

- Molecular catalysis

- Coordination polymers

- Chemistry of materials

- Organometallic and coordination chemistry for design of new materials

One session of the conference will be specially allocated for the young scientists.

Deadlines:

Registration - 15.03.2015; second circular - 15.03.2015; abstract submission (in electronic form) - 15.05.2015.

\section{Secretariat}

Dr. Klara G. Shalnova (Scientific Secretary), G. A. Razuvaev Institute of Organometallic Chemistry of Russian Academy of Sciences, 603950, GSP-445, 49, Tropinina str., Nizhny Novgorod, Russian Federation.

Tel.: +7 (831) 462 7682; Fax: +7 (831) 462 7497; E-mail: razuvaev2015@iomc.ras.ru
- Plenary lectures (40 min)

- Invited lectures (30 min)

- Oral communications (20 min)

- Young scientists section communications (15 min)

- Posters

www: iomc.ras.ru/razuvaev2015 